\title{
Nailfold videocapillaroscopy in antisynthetase syndrome
}

\author{
G. Cassone ${ }^{1,2,3}$, M. Sebastiani ${ }^{1}$, L. Cavagna ${ }^{4}$, K. Triantafyllias ${ }^{5}$, \\ V. Codullo ${ }^{4}$, C. Salvarani ${ }^{1,3}$, A. Manfredi ${ }^{1}$ \\ ${ }^{1}$ Rheumatology Unit, University of Modena and Reggio Emilia, Modena, Italy; \\ ${ }^{2}$ Clinical and Experimental Medicine PhD Program, University of Modena and Reggio Emilia, Modena, Italy; \\ ${ }^{3}$ Rheumatology Unit, IRCCS Arcispedale Santa Maria Nuova, Azienda Unità Sanitaria Locale-IRCCS \\ di Reggio Emilia, Reggio Emilia, Italy; ${ }^{4}$ Division of Rheumatology, University and IRCCS Policlinico \\ S. Matteo Foudation, Pavia, Italy; ${ }^{5}$ CCURA Rheumatology Clinics, Bad Kreuznach, Germany
}

\section{SUMMARY}

A 57-year-old woman with a diagnosis of antisynthetase syndrome (ASSD) underwent a nailfold videocapillaroscopy (NVC) showing a scleroderma pattern.

Alterations in capillary morphology have been reported in adults with inflammatory myositis (IM) but only recently have the differences in NVC findings between these two diseases been established.

ASSD is currently classified as a subset of IM, for which reason only a few studies in literature evaluate its specific hallmarks, showing nonspecific features of NVC in patients with polymyositis and dermatomyositis (DM) and antisynthetase antibodies.

To our knowledge, this is the first description of ASSD capillaroscopy features, and the first report of NVC in ASSD with evidence of scleroderma pattern.

Further studies are needed to define clearly frequency, typical features, and possible correlation with clinical and serological data of NVC changes in ASSD, differences between microangiopathy in ASSD and systemic sclerosis or DM.

Key words: Nailfold videocapillaroscopy; Inflammatory myositis; Antisynthetase syndrome; Raynaud's phenomenon.

57-year-old woman presented with a five-month history of dry cough, dyspnoea, weakness, Raynaud's phenomenon and arthralgias. On physical examination, we noted hyperkeratosis, scaling and fissuring of the fingers, digital clubbing, swollen joints and pulmonary crackles. Hematologic laboratory tests showed increase of CPK (873 U/L) and LDH (541 U/L). Anti-nuclear antibodies were positive (1:160 speckled pattern), with anti-Jo1 positivity.

She underwent pulmonary high-resolution computed tomography, showing interstitial lung disease (ILD). A diagnosis of antisynthetase syndrome (ASSD) was made.

A nailfold videocapillaroscopy (NVC) showed a scleroderma pattern (Figure 1), characterized by disorganization of the vascular array (panels A, D), reduction of capillary density (panels A, B, D), enlarged and giant capillaries (panels $\mathrm{B}$ ), microhe- morrhages (panels $\mathrm{C}, \mathrm{D})$ tortuosities and ramified capillaries (panels A, D).

Alterations in capillary morphology have been reported in adults with inflammatory myositis (IM), such as polymyositis (PM) and dermatomyositis (DM) (1-3), but only recently have the differences in NVC findings between these two diseases been established (1). NVC alterations are significantly more frequent and severe in DM patients, in which a scleroderma-like pattern was described.

ASSD is currently classified as a subset of IM, characterized by the presence of serum autoantibodies directed to aminoacyl transfer RNA synthetases (anti-tRNA) along with features of ILD and arthritis, other than myositis (4). For this reason, only few studies in literature evaluate its specific hallmarks, showing nonspecific features of NVC in patients with PM and DM and antisynthetase antibodies.
Corresponding author:

Giulia Cassone

Rheumatology Unit,

Dpt. of Internal Medicine, University of Modena

and Reggio Emilia, Policlinico di Modena Via del Pozzo, 71 - 41125 Modena, Italy E-mail: cassonegiu@gmail.com 


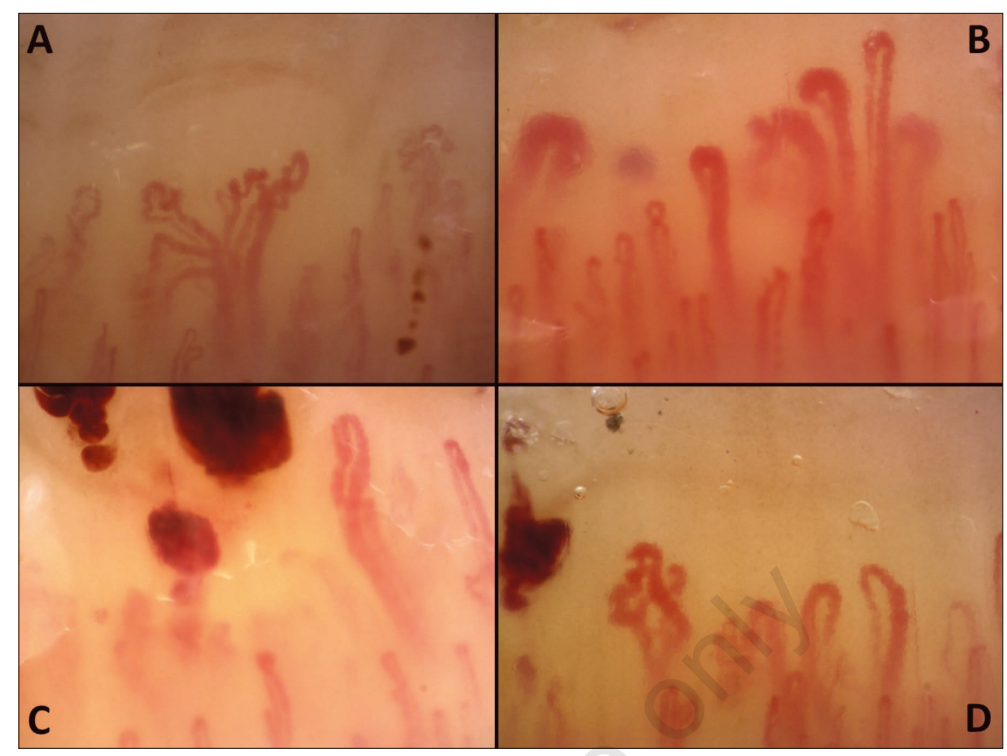

Figure 1 - Nailfold videocapillaroscopy showing a scleroderma pattern, characterized by disorganization of the vascular array $(A, D)$, reduction of capillary density $(A, B, D)$, enlarged and giant capillaries $(B)$, microhemorrhages $(C, D)$ tortuosities and ramified capillaries $(A, D)$.

\section{CONCLUSIONS}

To our knowledge, this is the first description of a scleroderma pattern at NVC in a patient with a diagnosis of ASSD.

Further studies are needed to define clearly frequency, typical features, and possible correlation with clinical and serological data of NVC changes in ASSD, differences between microangiopathy in ASSD and systemic sclerosis or DM.

Conflict of interest: the authors declare no conflict of interest.

\section{REFERENCES}

1. Manfredi A, Sebastiani M, Cassone G, et al. Nailfold capillaroscopic changes in dermatomyositis and polymyositis. Clin Rheumatol. 2015; 34: 279-84.

2. Selva-O'Callaghan A, Fonollosa-Pla V, Trallero-Araguás E, et al. Nailfold capillary microscopy in adults with inflammatory myopathy. Semin Arthritis Rheum. 2010; 39: 398-404.

3. Mercer LK, Moore TL, Chinoy H, et al. Quantitative nailfold video capillaroscopy in patients with idiopathic inflammatory myopathy. Rheumatology 2010; 49: 1699-705.

4. Monti S, Montecucco C, Cavagna L. Clinical spectrum of anti-Jo-1-associated disease. Curr Opin Rheumatol. 2017; 29: 612-7. 